\title{
Distressing Belching with Chorea Induced by Caudate Infarction
}

\author{
Yui Sanpei Akira Hanazono Sachiko Kamada Masashiro Sugawara \\ Department of Neurology, Akita University Graduate School of Medicine, Akita, Japan
}

\section{Keywords}

Belching $\cdot$ Chorea $\cdot$ Stroke $\cdot$ Caudate head $\cdot$ Sprouting

\begin{abstract}
Although belching is mostly associated with gastrointestinal disorders, it occasionally accompanies movement disorders such as Parkinsonism or dystonia. A woman in her 80 s presented distressing belching and chorea of the right arm and leg from 3 years earlier. A brain MRI showed a left caudate infarction and atrophic change. Haloperidol significantly improved belching and chorea. Caudate infarction can cause distressing belching with chorea. It might be important to select the appropriate drug by referring to the accompanying involuntary movement to treat belching with movement disorders.
\end{abstract}

\section{Introduction}

Belching is a rapid and complex movement involving different systems, such as the digestive tract and respiratory systems, which act to move air from the stomach to the oral cavity [1]. Although mostly associated with gastrointestinal disorders [2], it occasionally accompanies movement disorders such as Parkinson's disease [3], Huntington's disease [4], and neuroacanthocytosis [5]. Here, we report on a caudate infarction, which presented distressing belching with hemichorea.

\section{Case Report}

A woman in her 80 s gradually developed distressing belching and involuntary movement of the right arm and leg from 3 years earlier. She had nonerosive reflux disease 7 years ago, but medication immediately cured her symptoms. She also had senile dementia of Alzheimer 

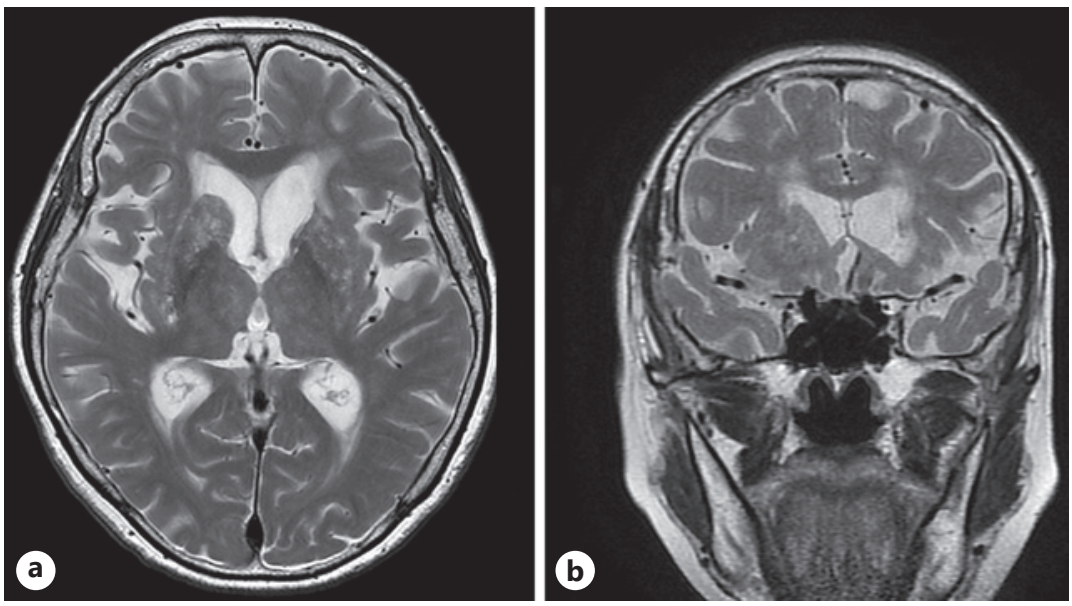

Fig. 1. Brain MRI of the patient. a, b T2WI. a, b Left head of caudate altered signal intensities and flattened.

type for 7 years. Her disorientation and amnesia had gradually progressed, but no symptoms and images suggestive of other dementias like Huntington's disease or Lewy body dementia were found. She had no history of diabetes mellitus and dyslipidemia and no family history with a neurodegenerative disease that involves basal ganglia. Upper gastrointestinal endoscopy could not reveal any organic abnormalities, so she referred to our department. Belching weakened when she was nervous (online suppl. Video 1; for all online suppl. material, see www.karger.com/doi/10.1159/000519762, Segment 1), and it was not seen during sleep or meals. The involuntary movement of the right arm and leg was rapid, brief, and irregular and could be suppressed voluntarily for short periods. We concluded that it was a chorea and no other movement disorder, such as Parkinsonism or dystonic movement, was observed. Routine laboratory studies revealed no abnormalities. A brain MRI showed chronic ischemic changes on bilateral basal ganglia and lacunar infarction of the left caudate (Fig. 1a, b). The atrophic change was observed only on the left side, not on the right side. Latency to the onset of symptoms after stroke was because of resilience and neuroplasticity which lead to aberrant network regeneration (sprouting). The latency period varied depending on stroke location, and the caudate was most involved in patients with symptoms within 6 months [6]. She showed only belching and chorea except for cognitive dysfunction of senile dementia of Alzheimer type. She was clinically diagnosed with belching and hemichorea caused by contralateral caudate infarction. She was treated with haloperidol $1.5 \mathrm{mg}$ per day. The reason for the choice was that the belching and chorea appeared at the same time and were thought to be related. After treatment, belching and chorea were significantly improved (online suppl. Video 1, Segment 2). More than 2 years have passed since the symptoms improved, but no deterioration of chorea and no other abnormal movements suggesting other basal ganglia diseases have appeared.

\section{Discussion}

This Present Case Provided Two Important Clinical Suggestions

First, caudate infarction could cause distressing belching with chorea. Chorea is the commonest movement disorder following stroke [7], and caudate infarction causes dystonia, chorea, and Parkinsonism [6]. However, there are no reports of belching induced by caudate infarction.

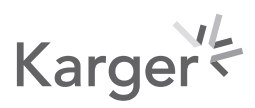


Previous reports of basal ganglia diseases had shown that Parkinsonism or dystonia had caused intractable belching in Parkinson's disease, Huntington's disease, and neuroacanthocytosis. Two patients of Parkinson's disease showed episodic belching during off periods [3], and fluoroscopic study revealed hypokinetic esophageal motility with air to enter the upper esophagus and to be retained because of disordered peristalsis. In these cases, apomorphine and domperidone (peripheral dopamine receptor blockade) relieved belching, and it was thought that disturbed central dopaminergic mechanisms affected laryngeal and pharyngeal muscles and the esophagus. A patient of Huntington's disease with marginal flattening of the caudate also showed repetitive belching and aerophagia [4], and a fluoroscopic study revealed periodic contractions of the diaphragm and irregular esophageal motility with air trapping. Low-dose trihexyphenidyl excellently improved the belching and aerophagia, and chorea responded to tetrabenazine. However, benzodiazepines, dopamine agonists including apomorphine, and valproate were ineffective. It had been concluded that this belching might be the result of upper gastrointestinal and diaphragmatic dystonia. Dystonic contractions of the upper airways and/or the diaphragm had been described in both primary and secondary dystonia [8]. In a patient of neuroacanthocytosis with belching [5], abdominal radiography showed air dilation of the entire gastrointestinal tract, and all-night polysomnography revealed irregular episodes of involuntary deep inspiration breathing followed by central apneas and then belching. Electromyography of the diaphragmatic muscle demonstrated repetitive recurrent arrhythmic and prolonged contractions of both diaphragmatic domes that can be characterized as dystonia. Several treatments failed to relieve symptoms, including antipsychotics, benzodiazepine, apomorphine, and levodopa, but trihexyphenidyl seemed not to be used.

Second, we need to select the appropriate drug by referring to the accompanying involuntary movement to treat belching with movement disorders. Before treatment, we were concerned that antipsychotics might exacerbate symptoms because Parkinsonism or dystonia was considered as a cause of belching in previous cases. However, in our case, belching appeared at the same period as chorea, and antipsychotic drug treatment improved both simultaneously, suggesting that chorea of the upper gastrointestinal tract and/or diaphragm may have been involved. This case showed that belching with basal ganglia disease can occur not only in Parkinsonism and dystonia but also in chorea and that the accompanying movement disorders may give suggestions to treatment choices.

\section{Statement of Ethics}

Written informed consent was obtained from the patient and family for publication of this case report and any accompanying images and data. The authors confirm that the paper is exempt from Ethical Committee approval because this work is a case report, and all information is fully anonymized.

\section{Conflict of Interest Statement}

The authors have no conflicts of interests to declare.

\section{Funding Sources}

No authors have received any funding.

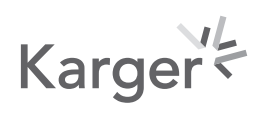




\section{Author Contributions}

Yui Sanpei wrote the first draft. Akira Hanazono, Sachiko Kamada, and Masashiro Sugawara contributed to review and critique.

\section{Data Availability Statement}

All data generated or analyzed during this study are included in this article and its online supplementary material files. Further inquiries can be directed to the corresponding author.

\section{References}

1 Lang IM. The physiology of eructation. Dysphagia. 2016;31(2):121-33.

2 Clearfield HR. Clinical intestinal gas syndromes. Prim Care. 1996 Sep;23(3):621-8.

3 Kempster PA, Lees AJ, Crichton P, Frankel JP, Shorvon P. Off-period belching due to a reversible disturbance of oesophageal motility in Parkinson's disease and its treatment with apomorphine. Mov Disord. 1989;4(1):47-52.

4 Hu MT, Chaudhuri KR. Repetitive belching, aerophagia, and torticollis in Huntington's disease: a case report. Mov Disord. 1998;13(2):363-5.

5 Sibon I, Ghorayeb I, Arné P, Tison F. Distressing belching and neuroacanthocytosis. Mov Disord. 2004;19(7): 856-9.

6 Suri R, Rodriguez-Porcel F, Donohue K, Jesse E, Lovera L, Dwivedi AK, et al. Post-stroke movement disorders: the clinical, neuroanatomic, and demographic portrait of 284 published cases. J Stroke Cerebrovasc Dis. 2018; 27(9):2388-97.

7 Alarcón F, Zijlmans JCM, Duañas G, Cevallo N. Post-stroke movement disorders: report of 56 patients. J Neurol Neurosurg Psychiatry. 2004;75(11):1568-74.

8 Braun N, Abd A, Baer J, Blitzer A, Stewart C, Brin M. Dyspnea in dystonia. A functional evaluation. Chest. 1995; 107(5):1309-16. 